\title{
Nutrition interventions by primary care staff: a survey of involvement, knowledge and attitude
}

\author{
Helen Moore ${ }^{1, *}$ and Ashley J Adamson ${ }^{2}$ \\ ${ }^{1}$ Centre for Research in Primary Care, University of Leeds, Hallas Wing, Nuffield Institute, 71-75 Clarendon Road, \\ Leeds LS2 9PL, UK: ${ }^{2}$ Human Nutrition Research Centre, University of Newcastle upon Tyne, UK
}

Submitted 19 September 2001: Accepted 17 December 2001

\begin{abstract}
Objective: To undertake a survey to investigate the quality and format of nutrition health promotion in UK primary care. Data from both primary care practitioners - i.e. level of nutritional knowledge and attitude towards nutrition interventions - and patients - i.e. the format and quality of dietary consultations - are presented.

Design: A self-completion questionnaire was used to assess nutritional knowledge and attitude of primary care staff towards nutrition interventions. Data regarding the format and quality of the dietary consultation were collected from patients using a screening question and follow-up questionnaire.

Setting: Twelve general practices in a city in north-east England.

Subjects: One hundred and nine primary care staff, and 2400 consecutive patients recruited from the 12 practices.

Results: Seventy-seven per cent of primary care staff completed the questionnaire. Sixty-five per cent of the knowledge questions were answered correctly by most practitioners. Questions containing complex nutritional terminology were answered poorly. Most practitioners believed primary care teams have an essential role in giving dietary advice. Thirteen per cent of patients reported that they had discussed diet. Of these, $40 \%$ were asked to make dietary changes; $20 \%$ discussed how they cooked or prepared food; and 33\% were asked to make a follow-up appointment.

Conclusion: Encouraging signs included good levels of nutritional knowledge and belief amongst staff that they should be involved in nutrition interventions. Patients reported that they understood and felt able to achieve the dietary changes suggested. Less encouraging were little evidence of discussion about the practical aspects of food and fairly low rates of follow-up being arranged.
\end{abstract}

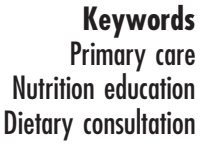

Keywords

Nutrition education Dietary consultation
The National Service Framework for Coronary Heart Disease (NSF) outlines a major health promotion remit for UK primary care ${ }^{1}$. Two of the standards related to health promotion are aimed directly at general practitioners (GPs) ${ }^{1}$ :

\section{'General Practitioners should:}

- Identify all people with established cardiovascular disease and offer them comprehensive advice and appropriate advice and treatment to reduce their risks.

- Identify all people at significant risk of cardiovascular disease but who have not yet developed symptoms and offer them appropriate advice and treatment to reduce their risks.'

This directive is not new; over the last 20 years successive governments made similar recommendations, central to their national health strategies. Surveys that assessed the extent of health-promoting activity in primary care in the late 1980 s and early 1990s reported 'disappointing' rates of involvement ${ }^{2,3}$. Several barriers have been highlighted that serve to limit any increase in involvement, including lack of time and confidence on behalf of practitioners ${ }^{4-6}$. The last decade has, however, seen a great increase in the number of practice nurses employed in primary care and health promotion is known to be a major part of their role ${ }^{7}$. This increase in personnel may mean that primary care can now meet the reported public demand for lifestyle advice provided by doctors and nurses ${ }^{3,8}$.

The role of diet in the development of cardiovascular disease (CVD) is well established; diet continues to be the cornerstone in the management of diabetes, the primary prevention of CVD and an important adjunct to drug

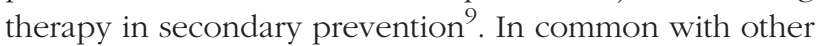
health-promoting activities, it has been reported that primary care staff are less than enthusiastic about their role as nutrition educators. Reported barriers to staff getting involved include lack of compliance on behalf of patients and a lack of professional satisfaction, particularly when advising patients about weight loss ${ }^{4,5}$. 
The level of enthusiasm of primary care staff, along with nutritional knowledge and ability to communicate this to patients, will have major implications for implementation of NSF standards. Previous surveys measuring nutritional knowledge amongst UK primary care staff reported broadly satisfactory levels of knowledge but with some significant gaps and difficulty with translation of knowledge into practical nutritional guidance for patients $5,10,11$.

This paper presents a survey of the readiness of primary care staff to carry out nutrition health promotion and information on the extent and format of such consultations in one city in the north east of England. The specific objectives of the study were:

- to measure the knowledge levels of primary care staff regarding diet and cardiovascular disease;

- to measure the attitude of primary care staff towards nutrition interventions;

- to measure the proportion of consultations in which diet was discussed; and

- to gather information on the format of dietary consultations.

\section{Method}

Data were collected in 1997 in Sunderland, an industrial city with a population of approximately 250000 . At the time of the survey, there were 53 GP practices within the Sunderland Health Authority area. All 53 practices were invited by letter to join the study. Of these, 18 practices expressed an interest and were visited by members of the research team. Twelve practices agreed to join the study.

\section{Measurement of the knowledge and attitude of primary care staff}

A self-completion questionnaire was used to assess (1) knowledge of diet and CVD and (2) attitude towards dietary interventions of the practice staff. The questionnaire was based on one used in a previous study ${ }^{12}$. Questions were adapted to reflect the most recent nutritional guidelines for the prevention of $\mathrm{CVD}^{9}$. Practices were asked to identify all members of their practice team who might be involved in giving nutritional advice to patients and questionnaires were given to these practitioners. From the 12 practices, 109 staff were identified and these included GPs, practice nurses, health visitors, district nurses and dietitians. Questionnaires were hand delivered to a previously identified key member of the practice team (usually the practice manager) and a date was arranged to collect them.

\section{Measurement of the proportion and format of dietary consultations}

Data on the format and quality of the dietary consultation were gathered from patients, using a screening question and follow-up questionnaire. These assessment tools were

\section{The Five-Stage Dietary Counselling Model}

1. Screening

2. Assessment

3. Discussion and negotiation

4. Goal setting

5. Monitoring

Fig. 1 The Five-Stage Dietary Counselling Model. Adapted from the Health Education Authority's 'Helping People Change' initiative, $1993^{13}$

developed by the research team and piloted in a neighbouring health authority. In each of the 12 practices 200 consecutive patients were asked whether they had 'discussed their diet, food or anything about what they eat', immediately after leaving any of the practice consulting rooms. The self-completion questionnaire was given to those patients who responded positively to the screening question. The questionnaire investigated the format of the dietary consultation. Questions were based around a model for dietary counselling, the Five-Stage Dietary Counselling Model (see Fig. 1) ${ }^{13}$. This model has not been formally evaluated but elements in the approach are drawn from patient-centred care, which has been shown to improve patient outcome ${ }^{14}$. Also, the model includes monitoring as a key element and this ongoing support has been shown to improve the likely success of lifestyle interventions, such as those for weight loss ${ }^{15,16}$. Questions were designed to explore the extent to which the various stages were present in dietary consultations.

To minimise the impact of data collection on the behaviour of practice staff in consultations, the research team negotiated a week during which they could collect data on practice premises and within that week data collection sessions were chosen at random. Staff were not initially aware when the research team would be present, although it is acknowledged that this may have become evident during the course of the data collection sessions.

\section{Results}

\section{Practitioner nutritional knowledge and attitude}

Eighty-four (77\%) completed questionnaires were returned. Thirty-three (39\%) of these were completed by GPs, 17 (20\%) by practice nurses, 11 (13\%) by health visitors, 16 (19\%) by district nurses, three (4\%) by midwives, one (1\%) by a student health visitor, one by a trainee GP and two by other staff. Fifty-four (65\%) of the respondents were female. 
Table 1 Nutrition knowledge survey: questions to which less than half of respondents gave the correct response $(n=84)$

\begin{tabular}{|c|c|c|}
\hline Question & $\begin{array}{l}\text { Correct } \\
\text { response }\end{array}$ & $\begin{array}{c}\text { Correct } \\
\text { responses } \\
(\%)\end{array}$ \\
\hline Eggs should be restricted to $\leq 2$ per week & False & 38 \\
\hline Red meat should be avoided when trying to reduce serum cholesterol & False & 29 \\
\hline Cholesterol in food is the most important dietary factor in controlling blood lipids & False & 34 \\
\hline Stopping smoking leads to weight gain which cancels out the benefit of smoking cessation & False & 34 \\
\hline According to government recommendations most adults should eat more non-starch polysaccharides & True & 31 \\
\hline A teaspoon of margarine contains less fat than a teaspoon of butter & False & 47 \\
\hline To meet HoN targets the average British diet needs to have a reduction in total fat equivalent to $8-10 \%$ & True & 31 \\
\hline
\end{tabular}

A high proportion (65\%) of the questions in the nutrition knowledge survey were answered correctly by most practitioners. However, around one-third (29\%) of the questions were answered poorly, with less than half of the respondents giving the correct response. These poorly answered questions are shown in Table 1. The question that attracted the lowest number of correct responses (29\%) concerned the avoidance of red meat in the management of blood cholesterol.

Table 2 shows practitioners' self-rated knowledge regarding specific nutritional topics. More generally, most practitioners (63\%) rated their nutritional knowledge as average but the majority of practitioners (76\%) also believed that their knowledge was fairly up-to-date. The majority of practitioners (83\%) believed that they were occasionally successful in helping patients make changes to their diet, whilst only $15 \%$ felt that they were usually successful and nobody believed that they were always successful.

There were no significant differences between professional groups in their nutrition knowledge scores, selfrated knowledge or perception of success in helping patients change their diet or the role of nutrition in primary care. There was a difference in self-perception of ability to advise on obesity management $(P=0.004)$, with GPs and health visitors being more likely than other groups to rate their ability as good; however, subgroups for this between-group analysis were small.

Table 3 shows practitioner attitudes towards the importance of nutrition in primary care. Whilst the majority of the sample agreed that nutrition has an important role to play in disease prevention (99\%) and that the primary care team has an essential role in provision of dietary advice (99\%), they also believed (76\%) that they have insufficient time to advise patients adequately.

Table 2 Self-rated nutritional knowledge of practice staff $(n=84)$

\begin{tabular}{lc}
\hline How would you rate your... & $\begin{array}{c}\text { Percentage } \\
\text { responding } \\
\text { good or excellent }\end{array}$ \\
\hline Personal knowledge of diet and CVD? & 32 \\
Personal knowledge of diet and diabetes? & 31 \\
Ability to advise on diet and obesity? & 37 \\
\hline
\end{tabular}

\section{Information regarding dietary consultations}

In total, 2400 patients, 200 from each of the 12 practices, were asked a screening question. Three hundred and five (13\%) of the patients screened stated that they had discussed diet during the consultation they had just left. Of these patients, 54 (18\%) refused to go on to complete a questionnaire, the main reason for refusal being lack of time. Two hundred and fifty-one (82\%) went on to complete a questionnaire. Sixty-five per cent of those completing a questionnaire were female.

Ninety-four (37\%) patients stated that the doctor had raised the subject of nutrition during the consultation. Fifty-six (22\%) stated that a nurse had raised the subject, whilst 74 (29\%) patients stated that they had raised the subject themselves. The remaining 10\% stated that the subject had been raised by another health professional.

Information regarding the format of the dietary consultation is shown in Table 4. The majority of patients (62\%) recalled being asked what kind of food they eat now and just less than half (40\%) were asked to make changes to what they eat. Of this subgroup of patients who were asked to make changes, the majority (67\%) recalled being involved in deciding which changes they would make. A larger majority felt that the advice about dietary change was useful (88\%) and that they understood what was being asked of them (99\%). Of the patients asked to make changes, 90\% felt they would be able to make them.

Twenty per cent of patients were asked about how they cooked or prepared food, and of those asked to make some change to their diet (103), 11\% were asked to make changes to how they cooked or prepared food.

Thirty-two per cent of patients recalled being asked to

Table 3 Self-reported attitude towards the importance of nutrition in primary care $(n=84)$

\begin{tabular}{lc}
\hline & $\begin{array}{c}\text { Percentage } \\
\text { agreeing } \\
\text { with statements }\end{array}$ \\
\hline $\begin{array}{c}\text { Nutrition has an important part to } \\
\text { play in the prevention of disease } \\
\begin{array}{c}\text { The primary care team has an } \\
\text { essential role in giving dietary advice } \\
\text { The primary care team has insufficient } \\
\text { time to advise patients adequately }\end{array}\end{array}$ & 99 \\
\begin{tabular}{l} 
Advice given will not impact on what people eat \\
\hline
\end{tabular} & 30 \\
\hline
\end{tabular}


Table 4 Information from patients regarding the dietary consultation

\begin{tabular}{lr}
\hline & $\begin{array}{c}\text { Number } \\
\text { of 'yes' } \\
\text { Question }\end{array}$ \\
\hline Were you asked about what kind of food you eat now? & $155 / 251(62)$ \\
Were you asked about how you prepare or cook food? & $49 / 251(20)$ \\
Were you asked to make any changes to what you eat? & $101 / 251(40)$ \\
Were you asked to make any changes to how you prepare or cook food? & $27 / 251(11)$ \\
Were you involved in deciding which changes to make? & $68 / 103(67)$ \\
Was the advice about the changes to what you eat helpful to you? & $91 / 103(88)$ \\
Were you given advice on how to make these changes? & $69 / 103(67)$ \\
Do you understand the changes you were asked to make? & $102 / 103(99)$ \\
Do you think you will be able to make the changes you were asked to make? & $93 / 103(90)$ \\
Today, were you given any leaflets to read about what you eat? & $29 / 251(12)$ \\
Have you been asked to make another appointment to talk about what you eat? & $81 / 251(32)$ \\
\hline
\end{tabular}

make another appointment to talk about what they eat. Of those, $59 \%$ were to come back to see the practice dietitian, $21 \%$ to see the nurse and $18 \%$ to see the doctor.

\section{Discussion}

The survey findings provide a picture of nutrition health promotion in UK primary care in the years prior to publication of the NSF. It provides information on how well equipped primary care practitioners were to provide dietary advice to patients. Reports from patients provide information about the format of dietary consultations.

\section{Reliability and validity of the data}

Several issues should be considered in the interpretation of this research. It is acknowledged that practices opting to join such a survey represent a motivated subset of the primary care population. In addition, using patient recall to gather information on the consultation does have some drawbacks. It is recognised that patients might forget some aspects of the consultation ${ }^{17}$. In this study this problem was minimised by asking about the consultation immediately after the patient left the consulting room. It is also recognised that, as for any questionnaire, respondents may wish to give socially desirable responses, thus overreporting recognised desirable behaviours ${ }^{18}$.

\section{Nutritional knowledge of primary care staff}

In keeping with other surveys of UK general practice staff, the results of the nutritional knowledge survey show that levels of basic nutritional knowledge are fairly good go,10,11. $^{5}$. In this survey, the majority of practice staff answered most of the questions correctly and this did not appear to differ across the professional groups. Most staff were clear that dietary recommendations for the general population advise a reduction in total dietary fat, an increase in starchy foods and an increase in fruit and vegetables. However, as reported in other UK surveys, there were some specific areas where a significant amount of confusion existed ${ }^{5,10}$. In particular, the area of lipidlowering diets and dietary fats appeared to cause confusion. Over half of the staff surveyed stated that egg consumption should be restricted to no more than two per week, a recommendation that can no longer be justified 9 . Around two-thirds of practitioners stated that red meat should be avoided when trying to lower serum cholesterol and almost half believed that dietary cholesterol was the most important dietary factor in controlling serum cholesterol.

Uncertainty increased where questions contained more complex nutritional terminology; for example, less than half of respondents gave the correct response to the questions containing the terms 'monounsaturates' and 'non-starch polysaccharides'. This apparent lack of knowledge or unfamiliarity with nutritional terms is potentially important, as primary care staff may well be involved in the interpretation of nutritional guidelines for patients.

\section{Self-perception and attitudes of primary care staff towards diet and dietary consultations}

The non-compliance of patients has been cited as a barrier to primary care staff becoming involved in dietary counselling and health promotion in both UK and international surveys ${ }^{5,19}$. In this survey, the vast majority of practitioners felt that they were only occasionally successful in helping patients to make dietary changes. However, around $70 \%$ of respondents believed that the advice they gave would have at least some impact on what people eat.

Despite the rather low expectation of a successful outcome, there was overwhelming endorsement of the importance of nutrition in disease prevention and only $1 \%$ of the staff disagreed with this. Respondents also strongly believed that lack of time prevented them from adequately advising patients about diet, which confirms findings of earlier work ${ }^{4}$.

\section{Information on the proportion and format of dietary consultations}

This survey estimates that diet was discussed in around $13 \%$ of consultations. This is slightly lower than levels that 
might be expected as estimated by Dutch workers who stated that nutrition guidance is an essential part of treatment for around 16\% of presented episodes of illness ${ }^{20}$. A 1992 UK survey of health-promoting activity in general practice estimated the rate of nutrition health promotion to be $12 \%^{2}$. If reflected nationally, the results of the Sunderland survey suggest only a slight increase in the amount of nutrition health promotion occurring in UK primary care in recent years.

Results of the patient survey provide both encouraging messages about the format of dietary consultations and some cause for concern. In most cases the issue of diet was initially raised by a health professional and in around 40\% this was a GP. However, in almost a third of cases, the patients themselves reported that they had raised the subject and this seems to endorse earlier surveys that suggest patients do want such advice from practitioners.

The results offered an encouraging sign that dietary advice was usually preceded by assessment of current intake. Further encouragement was provided by the fact that two-thirds of patients asked to make change felt that they had been involved in deciding what changes to make, providing some indication of patient-centred care. Similarly, patients felt that they had been given advice to help make the changes and that they understood what was being asked of them. Almost 90\% felt the advice was helpful and that they would be able to make the changes suggested.

Less encouraging was the low number of patients who had been engaged in discussions about practical aspects of changing their diet. Only a fifth of patients were asked about how they prepared or cooked food and only a tenth of patients were asked to make dietary changes specific to food preparation or cooking. This may suggest a medicalisation of food in the primary care setting. Failure to engage in discussion about the practical aspects of food preparation may inhibit the ability of patients to put advice into practice.

Interestingly, only $10 \%$ of patients were given written information to back-up verbal advice, which seems rather low. In a UK survey of management of weight problems, 93\% of GPs stated that they would use a diet sheet to educate patients ${ }^{4}$. The low number of patients reporting that they were given a diet sheet in our survey may be partly explained by the fact that the question was related only to the consultation they had just left. Patients may have been given written information in an earlier contact in the same episode of care.

\section{Conclusion}

Successive governments have promoted the role of primary care in the implementation of lifestyle modification with the aim of preventing the major chronic diseases of this century. The latest government initiative continues to assign primary care a key position in primary and secondary prevention. This study provides evidence that, in the run-up to publication of the NSF, primary care staff were providing dietary advice in more than one in 10 consultations and had a positive attitude towards it. However, it also highlighted areas where improvements to care were possible, including gaps in nutritional knowledge, consistent with those reported previously. These persistent areas of weakness represent a real barrier to delivery of effective nutrition guidance in the primary and secondary prevention of coronary heart disease. Government will need to provide support for ongoing education and possible re-organisation of service delivery, if it is to enable primary care staff to fulfil NSF criteria.

\section{Acknowledgements}

We would like to thank the General Practices and Department of Nutrition and Dietetics in Sunderland for their help with this project. In addition, we thank members of the project steering group, including Mrs Alison Cullen, Dr Colin Waine and Dr Tim Gill, for their advice and support.

\section{References}

1 Department of Health. National Service Framework for Coronary Heart Disease. Modern Standards and Service Models. Department of Health, London, 2000.

2 Silagy C, Muir J, et al. Lifestyle advice in general practice: rates recalled by patients. $\mathrm{Br}$. Med. J. 1992; 305: 871-4.

3 Wallace PG, Brenin PL, et al. Are general practitioners doing enough to promote healthy lifestyle? Findings of the Medical Research Council's general practice research framework study on lifestyle and health. Br. Med.J. 1987; 294: 940-2.

4 Cade J, O'Connell S. Management of weight problems and obesity: knowledge, attitudes and current practice of general practitioners. Br. J. Gen. Pract. 1991; 41: 147-50.

5 Buttriss JL. Food and nutrition: attitudes, beliefs and knowledge in the United Kingdom. Am. J. Clin. Nutr. 1997; 65(Suppl.): 1985S-95S.

6 Mant D. Effectiveness of dietary intervention in general practice. Am. J. Clin. Nutr. 1997; 65(Suppl.): 1933S-8S.

7 Atkin K, Hirst M, Lunt N, Parker G. The role and selfperceived training needs of nurses employed in general practice: observations from a national census of practice nurses in England and Wales. J. Adv. Nursing 1994; 20: $46-52$.

8 Gallagher M. Patients' views. Practice Nursing 1994; 15: $8-12$.

9 Department of Health, Committee on Medical Aspects of Food Policy. Nutritional Aspects of Cardiovascular Disease. Report on Health and Social Subjects No. 46, London: HMSO, 1994.

10 Francis J, Roche M. Would primary health care workers give appropriate dietary advice after cholesterol screening? $\mathrm{Br}$. Med.J. 1989; 298: 1620-2.

11 Murray OS, Narayan V, et al. Study of dietetic knowledge among members of the primary health care team. Br.J. Gen. Pract. 1993; 43: 229-31.

12 Cadman L, Wiles R. Nutrition advice in primary care: evaluation of practice nurse nutrition training programmes. J. Hum. Nutr. Diet 1996; 9: 147-56.

13 Health Education Authority (HEA). Helping People Change. HEA, London, 1993. 
14 Stewart M. Effective physician-patient communication and health outcomes: a review. Can. Med. Assoc. J. 1995; 152 $1423-33$.

15 NHS Centre for Reviews and Dissemination. The prevention and treatment of obesity. Effective Health Care 1997; 3(2): 4.

16 National Audit Office. Tackling Obesity in England. Report by the Comptroller and Auditor General No. HC220. National Audit Office, February 2001.

17 Ley P, Bradshaw PW, Eaves D, Walker CM. A method for increasing patients' recall of information presented by doctors. Psychol. Med. 1973; 3: 217-20.
18 Oppenheim AN. Questionnaire Design, Interviewing and Attitude Measurement. London: Pinter Publishers Limited, 1997; 140.

19 Kushner RF. Barriers to providing nutrition counselling by physicians: a survey of primary care practitioners. Prev. Med. 1995; 49(11): 842-51.

20 Van Weel C. Morbidity in family medicine: the potential for individual nutritional counselling, an analysis from the Nijmegan Continuous Morbidity Registration. Am. J. Clin. Nutr. 1997; 65(Suppl.): 1928S-32S. 